\title{
Arterial embolism in a patient with pulmonary embolism and patent foramen ovale
}

\author{
Waiel Abusnina, $\mathrm{MD}^{\mathrm{a}}$ (1), Mohammed Megri, $\mathrm{MD}^{\mathrm{b}}$, Basel Edris, $\mathrm{MD}^{\mathrm{c}}$, and Mehiar El-Hamdani, $\mathrm{MD}^{\mathrm{C}}$ (1) \\ aDepartment of Internal Medicine, Joan C. Edwards School of Medicine, Marshall University, Huntington, West Virginia; 'Division of \\ Pulmonary/Critical Care and Sleep Medicine, University of Kentucky Medical Center, Lexington, Kentucky; 'Department of Cardiovascular \\ Disease, Joan C. Edwards School of Medicine, Marshall University, Huntington, West Virginia
}

\begin{abstract}
Paradoxical embolization is an uncommon but devastating complication of pulmonary embolism and continues to be frequently missed. Although the prevalence of patent foramen ovale is $25 \%$ to $30 \%$, the risk of paradoxical embolism is $<2 \%$ of all arterial ischemia. Paradoxical embolism is infrequent but can involve almost any artery of the body. Here, we present a case of a 65-year-old woman with paradoxical systemic arterial embolism secondary to deep venous thrombosis and pulmonary embolism in the presence of patent foramen ovale. High suspicion for paradoxical embolism is needed in the event of unexplained arterial occlusion. Awareness of this complication with prompt recognition and treatment could serve to preclude significant disability and death.
\end{abstract}

KEYWORDS Atrial septal aneurysm; cryptogenic stroke; Eustachian valve; paradoxical embolism; patent foramen ovale; pulmonary embolism

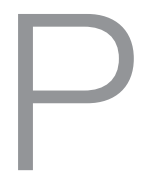

aradoxical arterial embolism may cause the first symptoms in patients with a coexisting hypercoagulable state and patent foramen ovale (PFO). This can result in significant morbidity and mortality depending on the location of the embolism. ${ }^{1}$ PFO occurs in about $25 \%$ of the general population, and most patients with $\mathrm{PFO}$ are asymptomatic. ${ }^{2} \mathrm{PFO}$, however, may be the underlying etiology of recurrent arterial embolic events, such as cryptogenic stroke or peripheral embolism. ${ }^{3}$ We present a case with paradoxical systemic arterial embolism secondary to deep venous thrombosis and pulmonary embolism (PE) in the presence of PFO.

\section{CASE PRESENTATION}

A 65-year-old nonsmoking woman with morbid obesity (body mass index $51 \mathrm{~kg} / \mathrm{m}^{2}$ ), with known uncontrolled insulin-dependent type 2 diabetes mellitus, controlled hypertension, hypothyroidism, and dyslipidemia presented to the emergency department complaining of numbness, tingling, and severe, aching right arm pain. She rated the pain 10 out of 10 in severity for the previous 2 hours. She also complained of acute, progressively worsening dyspnea.
Her family history was negative for any thromboembolic disorders.

On admission, her blood pressure was $128 / 78 \mathrm{~mm} \mathrm{Hg}$, heart rate 79 beats per minute, respiratory rate 20 breaths per minute, temperature $98.9^{\circ} \mathrm{F}$, and oxygen saturation $95 \%$ on room air. Cardiopulmonary examination was unremarkable, and extremity examination did not demonstrate any cyanosis or edema; however, her right upper limb radial, ulnar, and brachial pulses were nonpalpable and were noted to be cold and clammy. The rest of the exam was unremarkable.

A complete blood count, complete comprehensive panel, and coagulation profile were unremarkable (Table 1). A peripheral arterial angiogram showed acute right axillary artery thrombosis, which was treated with an EverFlex self-expanding $8 \times 40 \mathrm{~mm}$ stent to the right axillary artery, thrombectomy to the right brachial and axillary arteries, and tissue plasminogen activator infusion catheter for the ulnar artery thrombosis. She was started on clopidogrel and atorvastatin $80 \mathrm{mg}$. A chest radiograph did not show any process that could explain the acute episode. Transthoracic echocardiogram revealed an ejection fraction of $65 \%$. The right

Corresponding author: Waiel Abusnina, MD, Department of Internal Medicine, Joan C. Edwards School of Medicine, Marshall University, 6633 Country Club Dr., Huntington, WV 25701 (e-mail: dr.abusnina@gmail.com)

Color versions of one or more of the figures in this article can be found online at www.tandfonline.com/ubmc.

Received December 18, 2018; Revised January 24, 2019; Accepted January 28, 2019. 
Table 1. Results of a complete blood count, complete comprehensive panel, and thromboembolic disorder workup

\begin{tabular}{|c|c|c|}
\hline Test & Patient value & Normal value \\
\hline White blood count (cells $/ \mu \mathrm{L}$ ) & $5.2 \times 10^{3}$ & $4.8-10.8 \times 10^{3}$ \\
\hline Hemoglobin (g/dL) & 12.7 & $12-15$ \\
\hline Hematocrit & $37.7 \%$ & $36 \%-47 \%$ \\
\hline Platelets (platelet $/ \mu \mathrm{L}$ ) & $319 \times 10^{3}$ & $140-450 \times 10^{3}$ \\
\hline Mean corpuscular volume (fL/red cell) & 91.4 & $80-100$ \\
\hline Hemoglobin A1c & $13.3 \%$ & $4 \%-6 \%$ \\
\hline Homocysteine (mg/dL) & 17 & $<13$ \\
\hline Sodium (mmol/L) & 142 & $135-145$ \\
\hline Potassium (mmol/L) & 3.2 & $3.3-5.1$ \\
\hline Chloride (mmol/L) & 113 & $96-108$ \\
\hline Carbon dioxide (mmol/L) & 24 & $21-32$ \\
\hline Calcium (mg/dL) & 9.4 & $8.5-10.1$ \\
\hline Creatinine (mg/dL) & 0.7 & $0.6-1.1$ \\
\hline Blood urea nitrogen (mg/dL) & 12 & $7-22$ \\
\hline Factor V Leiden & Negative (no mutation) & Negative \\
\hline Factor II (G20210A) & Negative (no mutation) & Negative \\
\hline Antithrombin III antibodies & $114 \%$ & $77 \%-123 \%$ \\
\hline Protein C & $113 \%$ & $66 \%-129 \%$ \\
\hline Protein S & $94 \%$ & $55 \%-124 \%$ \\
\hline Anticardiolipin immunoglobulin G (U/mL) & $<9$ & $0-14$ \\
\hline Anticardiolipin immunoglobulin M (U/mL) & 9 & $0-12$ \\
\hline Anti-dsDNA antibodies (IU/mL) & $<1$ & $0-9$ \\
\hline MTHF gene mutation & Positive & Negative \\
\hline
\end{tabular}

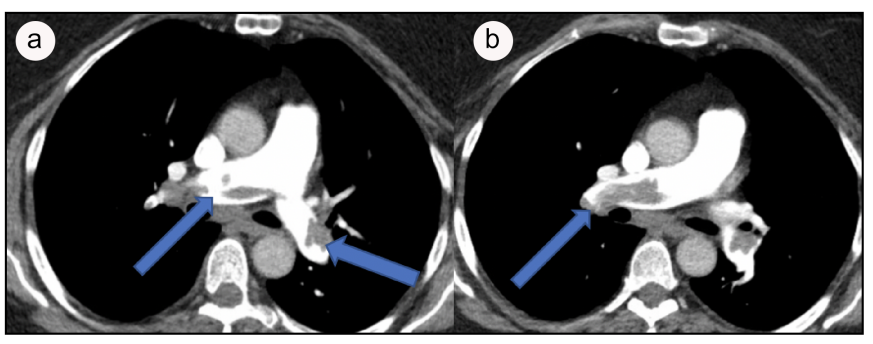

Figure 1. Saddle pulmonary embolism involving the right and left pulmonary arteries (blue arrows).

ventricle was moderately dilated with reduced ejection fraction, the right ventricular systolic pressure was 39 to $42 \mathrm{~mm}$ $\mathrm{Hg}$, an agitated saline study was positive with timing of bubbles consistent with intrapulmonary shunt, and no free-floating cardiac thrombi were detected. Computed tomography angiography showed a large saddle pulmonary embolus extending from the right and left main pulmonary arteries (Figure 1). Venous ultrasonography revealed a left-sided lower- limb deep venous thrombosis from the superficial femoral vein into the popliteal vein. The patient was started on a heparin drip and then bridged to apixaban. Factor V Leiden, Factor II, antithrombin III, Protein $\mathrm{C}$ and Protein S, anticardiolipin immunoglobulin G/immunoglobulin $\mathrm{M}$, and anti-dsDNA antibodies were all negative (Table 1). However, the MTHF mutation was present and coexistent with hyperhomocysteine.

Two weeks after discharge, arterial ultrasonography demonstrated normal right axillary artery flow. Transthoracic echocardiography showed improvement of the right ventricular function and a persistent, but small, PFO with a right-to-left shunt on bubble study. The patient had no more thrombotic events after 1 year of follow-up.

\section{DISCUSSION}

Paradoxical embolization is an uncommon but devastating complication of PE. Of all of the cases of systemic arterial emboli, the paradoxical arterial emboli have the lowest 
incidence but are frequently associated with cryptogenic stroke as well as peripheral embolism. ${ }^{4}$ Some patients with paradoxical embolism have a PFO, an atrial septal defect, or an atrial septal aneurysm. However, identification of one or more of these atrial septal abnormalities in a patient with an ischemic event does not prove a causal relationship because other sources or conduits of embolism may also be present. $^{5,6}$ Nonetheless, the likelihood of paradoxical embolism secondary to PFO increases with the presence of three important elements: a venous source of thrombus, a pulmonary embolus, and a right-to-left shunt through an intracardiac defect. ${ }^{7}$

Right-to-left shunt with atrial septal abnormalities could occur in any normal individual. Probable mechanisms include transient increases in right atrial pressure during early ventricular systole, use of the Valsalva maneuver, or repetitive cough, ${ }^{8}$ as well as increases in right ventricular pressure due to PE.

$\mathrm{PE}$ is the most common cause of acutely elevated right atrial pressure and right-to-left shunt in patients with PFO or atrial septal defect and occurs in at least $60 \%$ of paradoxical embolisms. ${ }^{9}$ Obstruction of $25 \%$ to $30 \%$ of the pulmonary vasculature is associated with pulmonary hypertension, although no patients experienced a mean pulmonary arterial pressure over $40 \mathrm{~mm} \mathrm{Hg}$ despite massive embolic obstruction. The right atrial pressure is elevated only occasionally for mean pulmonary arterial pressure $<30 \mathrm{~mm} \mathrm{Hg}$ but is increased consistently for mean pulmonary arterial pressure $>30 \mathrm{~mm} \mathrm{Hg}$. ${ }^{10,11}$

In our case report, the patient had the four main elements for diagnosis of paradoxical arterial embolism. She had a deep venous thrombosis, an acute saddle pulmonary embolism, a right ventricular systolic pressure of 39 to $42 \mathrm{~mm} \mathrm{Hg}$, and a positive agitated saline study, with timing of bubbles consistent with intrapulmonary shunt. All of these incidents increase the likelihood that her acute arterial thrombus was secondary to paradoxical embolism.
ORCID

Waiel Abusnina (iD http://orcid.org/0000-0003-2617-8483

Mehiar El-Hamdani (D) http://orcid.org/0000-0003-0066-9997

1. Guo S, Roberts I, Missri J. Paradoxical embolism, deep vein thrombosis, pulmonary embolism in a patient with patent foramen ovale: a case report. J Med Case Rep. 2007;1:104. doi:10.1186/1752-1947-1104 .

2. Hagen PT, Scholz DG, Edwards WD. Incidence and size of patent foramen ovale during the first 10 decades of life: an autopsy study of 965 normal hearts. Mayo Clin Proc. 1984;59:17-20.

3. Landzberg MJ, Khairy P. Indications for the closure of patent foramen ovale. Heart. 2004;90:219-224.

4. Foster PP, Boriek AM, Butler BD, Gernhardt ML, Bové AA. Patent foramen ovale and paradoxical systemic embolism: a bibliographic review. Aviat Space Environ Med. 2003;74(6 Pt 2):B1-64.

5. Harvey JR, Teague SM, Anderson JL, Voyles WF, Thadani U. Clinically silent atrial septal defects with evidence for cerebral embolization. Ann Intern Med. 1986;105:695-697.

6. Lamy C, Giannesini C, Zuber M, et al. Clinical and imaging findings in cryptogenic stroke patients with and without patent foramen ovale: the PFO-ASA Study. Stroke. 2002;33:706-711.

7. Johnson BI. Paradoxical embolism. J Clin Pathol. 1951;4:316-332.

8. Langholz D, Louie EK, Konstadt SN, Rao TL, Scanlon PJ. Transesophageal echocardiographic demonstration of distinct mechanisms for right to left shunting across a patent foramen ovale in the absence of pulmonary hypertension. J Am Coll Cardiol. 1991;18: 1112-1117.

9. Loscalzo J. Paradoxical embolism: clinical presentation, diagnostic strategies, and therapeutic options. Am Heart J. 1986;112:141-145.

10. Jardin F, Dubourg O, Guéret P, Delorme G, Bourdarias JP. Quantitative two-dimensional echocardiography in massive pulmonary embolism: emphasis on ventricular interdependence and leftward septal displacement. J Am Coll Cardiol. 1987;10:1201-1206. doi: 10.1016/S0735-1097(87)80119-5.

11. Dalen JE, Banas JS, Brooks HL, Evans GL, Paraskos JA, Dexter L. Resolution rate of acute pulmonary embolism in man. $N$ Engl J Med. 1969;280:1194-1199. doi:10.1056/NEJM196905292802202. 